ARTICLE

\title{
A rationally enhanced red fluorescent protein expands the utility of FRET biosensors
}

\author{
Gary C. H. Mo ${ }^{1,5}$, Clara Posner (10) ${ }^{1,2}$, Erik A. Rodriguez (10 ${ }^{3}$, Tengqian Sun ${ }^{1} \&$ Jin Zhang $\mathbb{B}^{1,2,4 凶}$
}

Genetically encoded Förster Resonance Energy Transfer (FRET)-based biosensors are powerful tools to illuminate spatiotemporal regulation of cell signaling in living cells, but the utility of the red spectrum for biosensing was limited due to a lack of bright and stable red fluorescent proteins. Here, we rationally improve the photophysical characteristics of the coralderived fluorescent protein TagRFP-T. We show that a new single-residue mutant, superTagRFP (stagRFP) has nearly twice the molecular brightness of TagRFP-T and negligible photoactivation. stagRFP facilitates significant improvements on multiple green-red biosensors as a FRET acceptor and is an efficient FRET donor that supports red/far-red FRET biosensing. Capitalizing on the ability of stagRFP to couple with multiple FRET partners, we develop a novel multiplex method to examine the confluence of signaling activities from three kinases simultaneously in single living cells, providing evidence for a role of Src family kinases in regulating growth factor induced Akt and ERK activities.

\footnotetext{
${ }^{1}$ Department of Pharmacology, University of California San Diego, La Jolla, CA 92093, USA. ${ }^{2}$ Department of Bioengineering, University of California San Diego, La Jolla, CA 92093, USA. ${ }^{3}$ Department of Chemistry, The George Washington University, Washington, DC 20052, USA. ${ }^{4}$ Department of Chemistry and Biochemistry, University of California San Diego, La Jolla, CA 92093, USA. ${ }^{5}$ Present address: Department of Pharmacology, University of Illinois at Chicago, Chicago, IL 60612, USA. ${ }^{凶}$ email: jzhang32@ucsd.edu
} 
T he coral proteins TagRFP ${ }^{1}$ and a single residue mutant TagRFP-T ${ }^{2}$ are red fluorescent proteins (RFPs) that are twice as bright as the extensively used RFP mCherry. Despite high biophysical brightness and well-characterized chromophore mechanics, TagRFP and TagRFP-T are underutilized due to high propensity for dark-state conversion ${ }^{3}$. This process causes fluorescence "blinking", which is undesirable in conventional fluorescence microscopy. We showed that through a phenomenon called Fluorescence fluctuation Increase by Contact (FLINC), the fluorescent protein Dronpa can specifically and quantitatively enhance the rate and probability of dark-state conversion of TagRFP-T. By controlling these fluctuations using conformational switches, we built a new class of fluorescent biosensors capable of biochemical activity imaging at superresolution ${ }^{4}$ in live cells. This discovery has a noteworthy corollary: in the absence of dark-state processes such as FLINC, TagRFP-T could become brighter, more stable, and better suited for conventional microscopy modes, such as FRET biosensing. Our previous work revealed that FLINC is mediated by external residues on TagRFP-T. Therefore, we chose not to target the internal residues in contact with the chromophore, like prior fluorescent protein optimizations. In this communication, we demonstrate how rational re-engineering of solvent-exposed residues leads to a highly useful RFP with improved stability and brightness that enhances FRET sensing and multiplexed imaging. We thus showcase green-red FRET biosensors with a dynamic range comparable to that of cyan-yellow FRET biosensors and reveal the complex interaction between the kinases Src, Akt and ERK within the same single cell.

\section{Results}

TagRFP-T is improved by mutagenesis at an external residue. Mutagenesis confirmed positively-charged residues on Dronpa mediate FLINC by electrostatic interactions, using a fusion model where Dronpa is directly fused to TagRFP-T ${ }^{4}$. We hypothesized that removing acidic residues on the exterior of TagRFP-T would reduce FLINC and dark-state conversion to produce a more desirable RFP. The Dronpa-TagRFP-T fusion model provides a quantitative means for mutagenic screening, in which an improved TagRFP-T mutant will decrease dark-state conversion and increase red fluorescence intensity compared to the wild-type TagRFP-T (Fig. 1a). We performed random mutagenesis at 4 solvent-exposed, charged residues on TagRFP-T fused to Dronpa. Two acidic residues D159 and D196 were expected to decrease dark-state conversion, while two basic residues (R157 and R198) were chosen as the negative controls and should not change the blinking or increase fluorescence intensity. Libraries of single and multiple site mutations were generated using fully degenerate primers, protein was expressed in E. coli, and the RFP fluorescence intensity was quantified. After 4 rounds of screening, fluorescence intensity increase was not observed at residues R157, R198, or D196, suggesting these external positions do not contribute significantly to TagRFP-T fluorescence and/or dark-state conversion. However, several mutations at D159 yielded significant improvements. Consistent with our hypothesis, basic and bulky hydrophobic mutations (His, Phe, and Val) at D159 increased red fluorescence intensity, creating fusion proteins that are brighter than TagRFP-T alone. One mutant, D159V, was the most prevalent ( $>50 \%$ probability in 2 rounds of random mutagenesis). D159V retained bright fluorescence when expressed alone and not fused to Dronpa. It was named super-TagRFP (stagRFP) and further characterized.

Organized Smooth Endoplasmic Reticulum (OSER) assays indicated that stagRFP has a tendency to dimerize or oligomerize (Supplementary Fig. 1), similar to parent proteins TagRFP and
TagRFP-T $T^{5,6}$. stagRFP contains the same number of tryptophan, tyrosine, and cysteine residues and same $\beta$-barrel fold as TagRFPT. We thus examined freshly purified TagRFP-T and stagRFP and normalized the absorbance at $280 \mathrm{~nm}$ to directly compare their absorption spectra. While stagRFP absorption and fluorescence spectra remain similar to that of TagRFP-T, the extinction coefficient and fluorescence quantum yield of stagRFP were larger (Fig. 1b, c). Published TagRFP-T extinction coefficients ${ }^{2}$ allowed us to determine stagRFP extinction coefficient as $113,000 \mathrm{M}^{-1} \mathrm{~cm}^{-1}$ and a quantum yield of 0.53 , resulting in an apparent brightness index (extinction coefficient $\times$ quantum yield) of 59,000. Using plasma membrane targeted TagRFP, TagRFP-T, and stagRFP expressed in HeLa cells, we further examined the stability of stagRFP. TagRFP-T and TagRFP are known to display significant and irreversible photoactivation, especially using wideband and blue-shifted excitation wavelengths used for GFP ${ }^{3}$. By using ratios of the stable red fluorescence intensity within each cycle of alternate excitation $\left(\mathrm{I}_{\text {after-GFP }} / \mathrm{I}_{\text {before-GFP }}\right)$, we quantified the irreversible photoactivation of these FPs (Fig. 1d). Both parent proteins showed significant photoactivation. TagRFP is significantly and fully activated after a few cycles, while the apparent intensity of TagRFP-T continues to increase even after 10 cycles. stagRFP showed negligible photoactivation.

stagRFP enhances FRET biosensing in the red spectrum. The green-red regions of the visible spectrum are favored for fluorescence imaging due to widely available excitation sources and relatively clean spectral separation. However, green-red genetically encoded FRET biosensors tend to have low dynamic range and are further complicated by the red chromophore maturation from green to red. The enhanced properties of stagRFP prompted us to examine whether it would facilitate a significant gain in dynamic range. We compared the performance of stagRFP as a FRET acceptor with commonly used RFPs in the context of GFPRFP based A Kinase Activity Reporters (AKARs) ${ }^{7}$. First, we show the control biosensor that incorporated wild-type TagRFP-T displayed a complex ratiometric response, where the acceptordirect fluorescence intensity steadily increased by over $50 \%$ due to TagRFP-T photoactivation and biased the ratiometric readout (Supplementary Fig. 2A). Substitution of TagRFP-T with stagRFP corrected this photoactivation artifact, making ratiometric FRET imaging straightforward (Supplementary Fig. 2B). The direct RFP fluorescence intensity in the stagRFP-based biosensor was stable and typically within $5 \%$ of initial intensity after more than $30 \mathrm{~min}$ of wide-field epifluorescence FRET imaging.

In the development of cyan-yellow FRET biosensors, such as AKAR, further improvements were made by introducing a brighter FRET donor ${ }^{8}$ or a semi-flexible linker ${ }^{9}$. We therefore utilized different GFPs and examined whether the incorporation of a more efficient donor and an additional linker could further improve the response. Comparing the responses of several biosensor variants upon stimulation of PKA using forskolin (Fsk) and 3-isobutyl-1-methylxanthine (IBMX), stagRFP enabled a significant increase in dynamic range. The average response of PKA biosensors using stagRFP was significantly larger than the same biosensor with mCherry. This is true for the AKAR biosensor: (1) with an EGFP donor and short-linker, where stagRFP enabled a $19.5 \pm 0.8 \%$ (mean \pm SEM; $n=17$ biologically independent cells) average response compared to $17.2 \pm 0.8 \%$ $(n=38)$ using mCherry $(p=0.04, t=2.080$, df $=47$ from two tailed, unpaired Welch's $t$-test); or (2) using a mClover donor and semi-flexible linker, where stagRFP achieved an average response of $39.1 \pm 2.1 \%(n=35)$ compared to $27.5 \pm 2.9 \%(n=6)$ using mCherry $(p=0.009, t=3.239 \mathrm{df}=10) \quad$ (Fig. 2a). The best performing biosensor was named GR-AKAR3, with EGFP and 


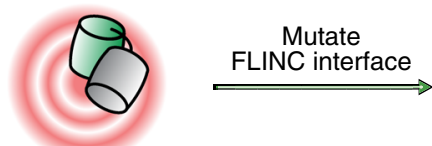

Unstable fluorescence

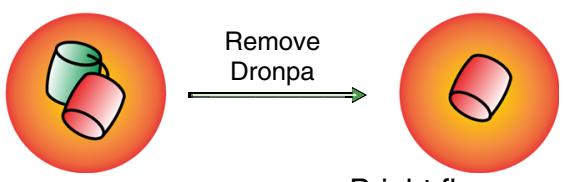

Bright fluorescence
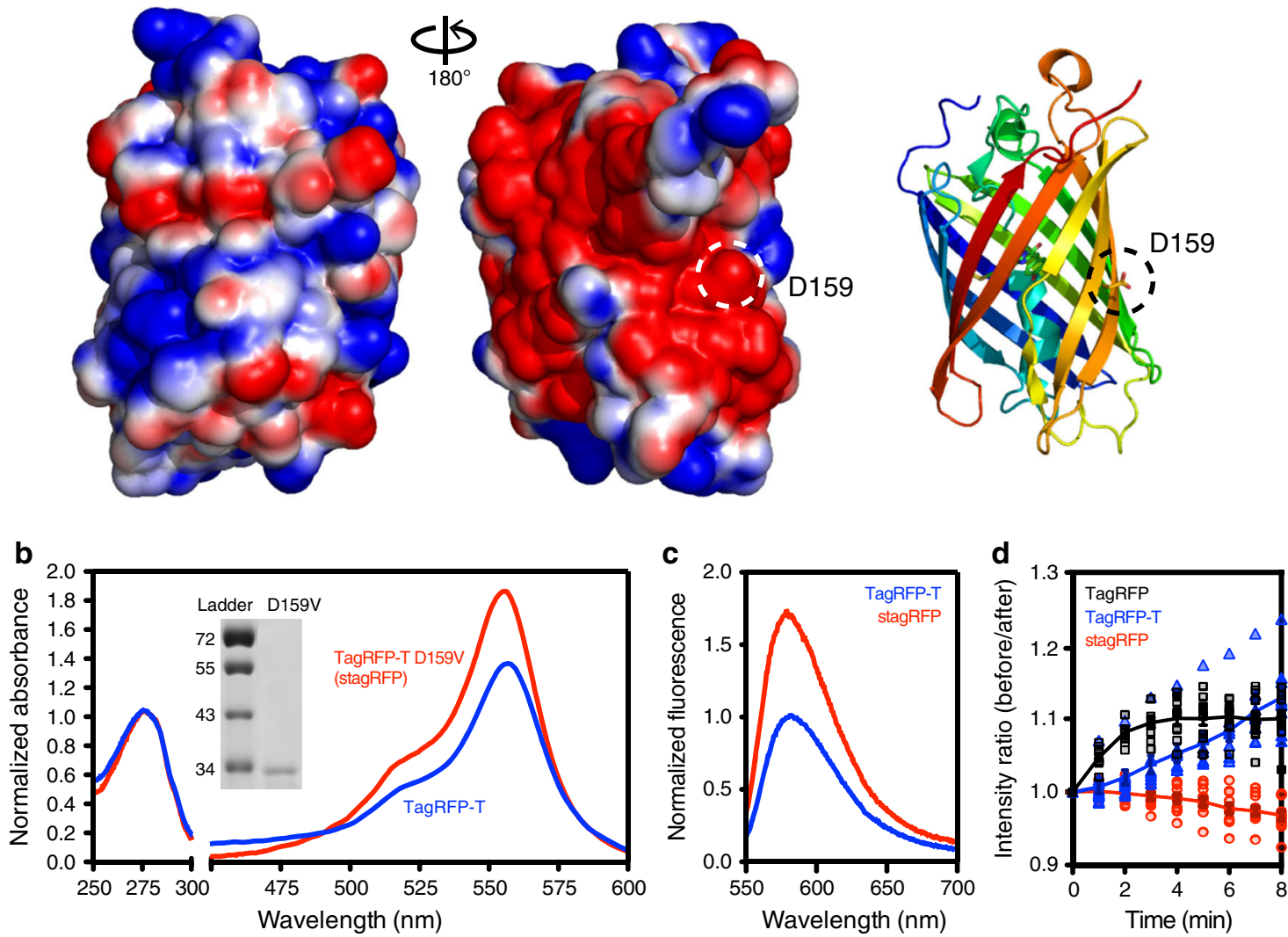

Fig. 1 The rational mutagenesis model and the resulting enhanced spectroscopic characteristics of stagRFP compared to TagRFP-T. a Schematic underlying the rational engineering approach. A model system bearing high FLINC state reveals the presence of the external acidic residue that weakens the TagRFP-T chromophore. Site-directed mutagenesis products that rescue the model back to a low FLINC state harbor mutants with improved insulation and stable fluorescence. Finally, removal of Dronpa confirms the improved behavior of the mutant TagRFP-T. A ribbon structure and APBS electrostatic surface of TagRFP-T (based on PDB 3T6H) demonstrates the large acidic (red) solvent exposed surface opposite the chromophore. The location of D159 is indicated by dashed circles. Normalized in vitro absorption (b) and fluorescence (c) spectra of purified stagRFP compared to TagRFP-T demonstrating the significantly higher extinction coefficient and quantum yield. $\mathbf{d}$ Cyclic excitation of HeLa cells expressing different TagRFP variants show photoactivation is present in parent proteins (TagRFP and TagRFP-T), but not the new mutant stagRFP (TagRFP, $n=9$ biologically independent cells; TagRFP-T, $n=10 ;$ and stagRFP, $n=12$ biologically independent cells); each cell is represented by the, respectively, colored symbol, and the average values across all cells are shown using solid lines. Source data for $\mathbf{b}-\mathbf{c}$ are provided as a Source Data file.

stagRFP as the FRET pair and a semi-flexible linker (AKARev), displayed a further improvement resulting in an average response of $44.6 \pm 2.1 \%(n=37$, maximum dynamic range of $80 \%)$ that is 6 -fold better than the average response of $8.1 \pm 0.9 \%(n=32)$ of the current best green-red biosensor AKAR2-CR ${ }^{10}$ utilizing the FRET pair mClover and mRuby, in the same cell lines (Fig. 2a). The new green-red GR-AKAR3 is comparable to the cyan-yellow PKA activity reporter AKAR4 ${ }^{8}$.

We further compared the performance of stagRFP in greenred FRET-biosensing with the recently developed ultra-bright red fluorescent protein mScarlet ${ }^{11}$ in this optimized AKARev format, replacing the stagRFP acceptor in GR-AKAR3 with mScarlet. Both stagRFP and mScarlet enabled robust detection of PKA activity, with GR-AKAR3 being slightly better-performing ( $p=$ $0.02, t=2.349, \mathrm{df}=55)$ than the mScarlet-based sensor $(38.0 \pm$ $1.8 \%, n=21)$. GR-AKAR3 is reversible like the predecessor AKAR4 (Fig. 2b). When targeted to the plasma membrane, the average response of Lyn-GR-AKAR3 is $16.7 \pm 1.5 \%$ (Fig. $2 \mathrm{c}, n=$ 20 , maximum response of $32 \%$ ) and comparable to that of the membrane-targeted, cyan-yellow PKA activity reporter LynAKAR4 ${ }^{8}$. In addition, the response of GR-AKAR3 can also be detected as a decrease in the mean donor (GFP) lifetime using Fluorescent Lifetime Imaging Microscopy (FLIM) (Fig. 2d) and as a decrease in the apparent mean acceptor fluorescence anisotropy using anisotropy imaging (Supplementary Fig. 3). Thus, stagRFP should facilitate the development of green-red FRET biosensors whose performance is on par with their cyanyellow counterparts.

We next examined the performance of stagRFP in second messenger cAMP biosensors, starting with the molecular switch within Epac-camps ${ }^{12}$, which is known to have a limited conformational change due to the use of a single cAMP binding domain of Epac1 instead of the full-length protein ${ }^{13}$. We made Epac1-camps biosensors that utilized mScarlet or stagRFP as 
a

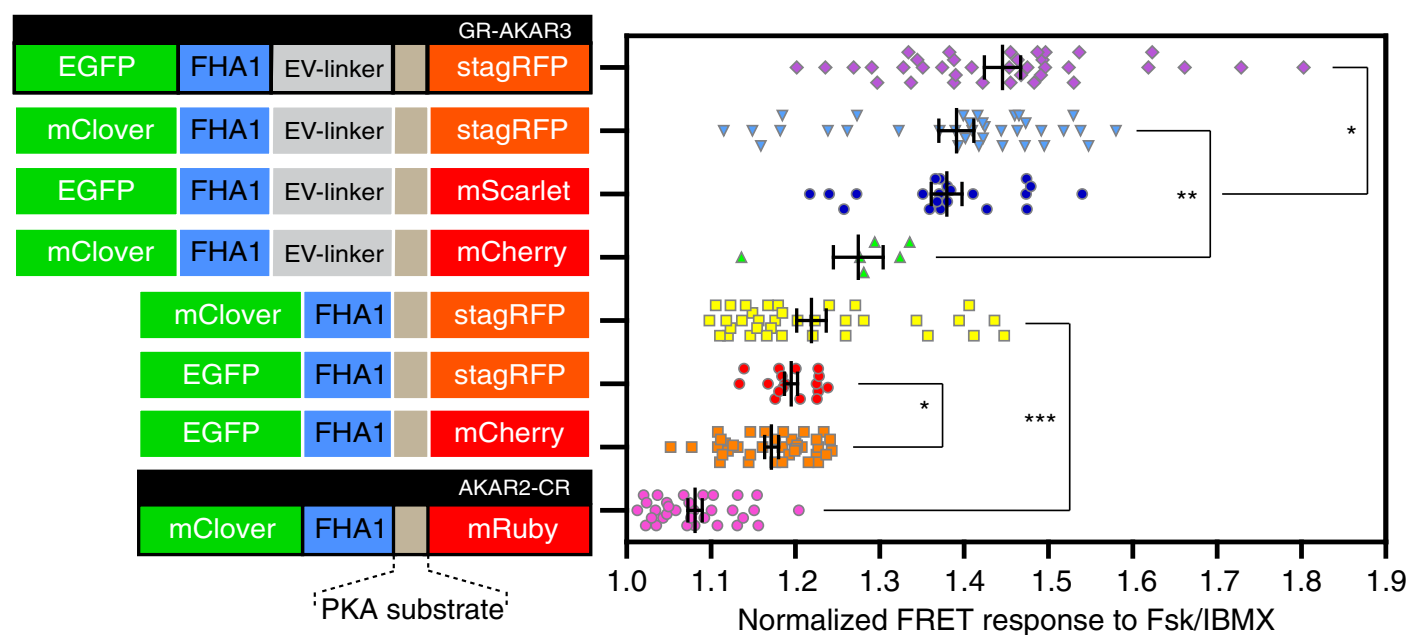

b

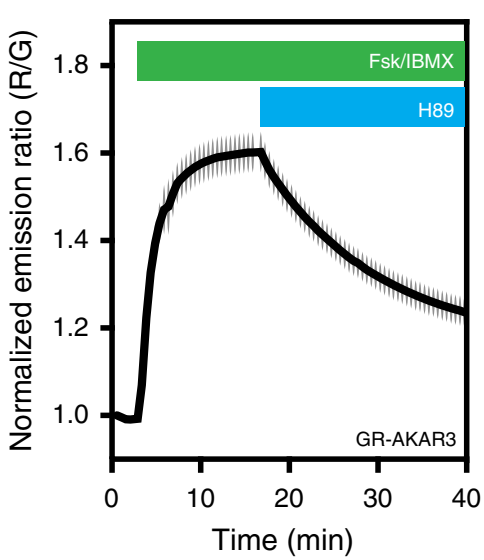

C

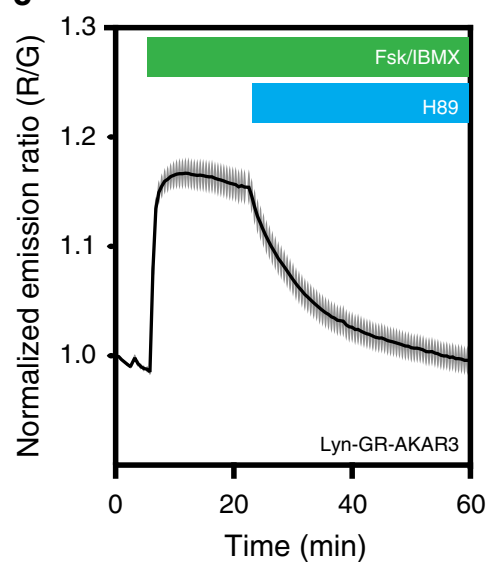

d

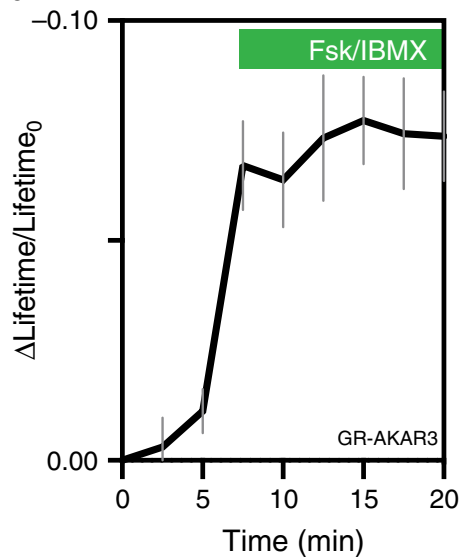

Fig. 2 Comparison highlighting the improved dynamic range of green-red A Kinase Activity Reporters (AKARs) when utilizing stagRFP. a stagRFP confers a significant increase in FRET dynamic range. The mean \pm SEM is displayed. Stimulation of PKA in HeLa or HEK293T cells was performed using $50 \mu \mathrm{M}$ forskolin and $100 \mu \mathrm{M}$ IBMX, while inhibition was performed using $20 \mu \mathrm{M} \mathrm{H} 89 .{ }^{\star} p<0.05 ;{ }^{\star \star} p<0.01 ;{ }^{\star \star \star} p<0.0001$ from two tailed, unpaired Welch's $t$-test. Specifically, $p$ values for the comparison between stagRFP and mScarlet in the AKARev context $p=0.0225(t=2.349$, df $=55)$; between stagRFP and mCherry in the mClover-AKARev context $p=0.0089(t=3.239, \mathrm{df}=10)$; between stagRFP and mCherry in the EGFP-AKAR context $p=$ $0.0430(t=2.080, \mathrm{df}=47)$; and between stagRFP and mRuby in the AKAR context $p<0.0001(t=15.80, \mathrm{df}=47)$. The changes in normalized emission ratio (red/green) are AKAR2-CR, $8.1 \pm 0.8 \%, n=32$ biologically independent cells; EGFP-mCherry-AKAR, 17.2 $\pm 0.8 \%, n=38 ;$ EGFP-stagRFP-AKAR, $19.5 \pm 0.7 \%, n=17$; mClover-stagRFP-AKAR, $21.9 \pm 1.7 \%, n=35 ;$ mClover-mCherry-AKARev, $27.5 \pm 2.9 \%, n=6 ;$ EGFP-mScarlet-AKARev, $38 \pm 1.8 \%, n=$ 21; mClover-stagRFP-AKARev, $39.1 \pm 2.0 \%, n=35$; EGFP-stagRFP-AKARev, $44.6 \pm 2.1 \%, n=37$. b Diffusible GR-AKAR3 displays a high dynamic range $(n=8)$. The mean ratio \pm SEM is displayed. Source data are provided as a Source Data file. $\mathbf{c}$ Membrane-targeted biosensor is able to detect the increase in membrane PKA activity $(n=20)$. The mean ratio \pm SEM is displayed. $\mathbf{d}$ The change in mean donor lifetime normalized to initial donor lifetime of GRAKAR3 in HeLa cells upon Fsk/IBMX stimulation $(n=6)$. The mean ratio \pm SEM is displayed in all panels.

acceptors at the C-terminal. In this context, the mScarlet-based biosensor showed minimal response $(0.3 \pm 0.2 \%, n=11)$, whereas the stagRFP counterpart showed a clearly significant FRET response $(3.3 \pm 0.4 \%, n=8, p=0.005, t=3.642$, $\mathrm{df}=9)$ (Fig. 3a). Despite the limited dynamic range, this data demonstrates that the use of stagRFP in biosensors could help identify functional molecular switches. We next incorporated stagRFP into a biosensor that utilizes truncated Epacl as the cAMP-dependent molecular switch to create a green-red variant of the cAMP biosensor ICUE ${ }^{14}$. Stimulation of HEK293T cells expressing the new GR-ICUE2 with isoproterenol (ISO), an adrenergic receptor agonist, showed that GR-ICUE2 could reversibly detect transient cAMP response (Fig. 3b). GR-ICUE2 displays an average FRET response of $60.5 \pm 3.0 \%(n=14$; maximum dynamic range of $71.0 \%)$ in HeLa cells upon Fsk and IBMX stimulation, which is close to the best cyan-yellow cAMP biosensor ICUE3 (average response $75.1 \pm 5.3 \%, n=12$; Fig. $3 \mathrm{c}$ top panel). The kinetics of cAMP increase upon Fsk and IBMX stimulation was also comparable to the currently most optimized version ICUE3 ${ }^{15}$ (Fig. 3c, lower panel).

The far-red and near-infrared portion of the visible spectrum was off-limits to FRET biosensing due to the lack of effective candidates for both an RFP donor and far-red acceptor. Recently, efforts have been made in creating FRET biosensors using two near-infrared FPs ${ }^{16}$. We note that FRET between an RFP and a far-red FP could prove advantageous for simultaneously imaging multiple sensors. A new bright, far-red FP incorporating the exogenous chromophore biliverdin and named the small UltraRed Fluorescent Protein (smURFP) ${ }^{17}$ should be useful as a FRET acceptor due to its large extinction coefficient of $180,000 \mathrm{M}^{-1} \mathrm{~cm}^{-1}$. We demonstrate that stagRFP is an effective donor to the smURFP acceptor on an ERK biosensor scaffold ${ }^{18}$. 

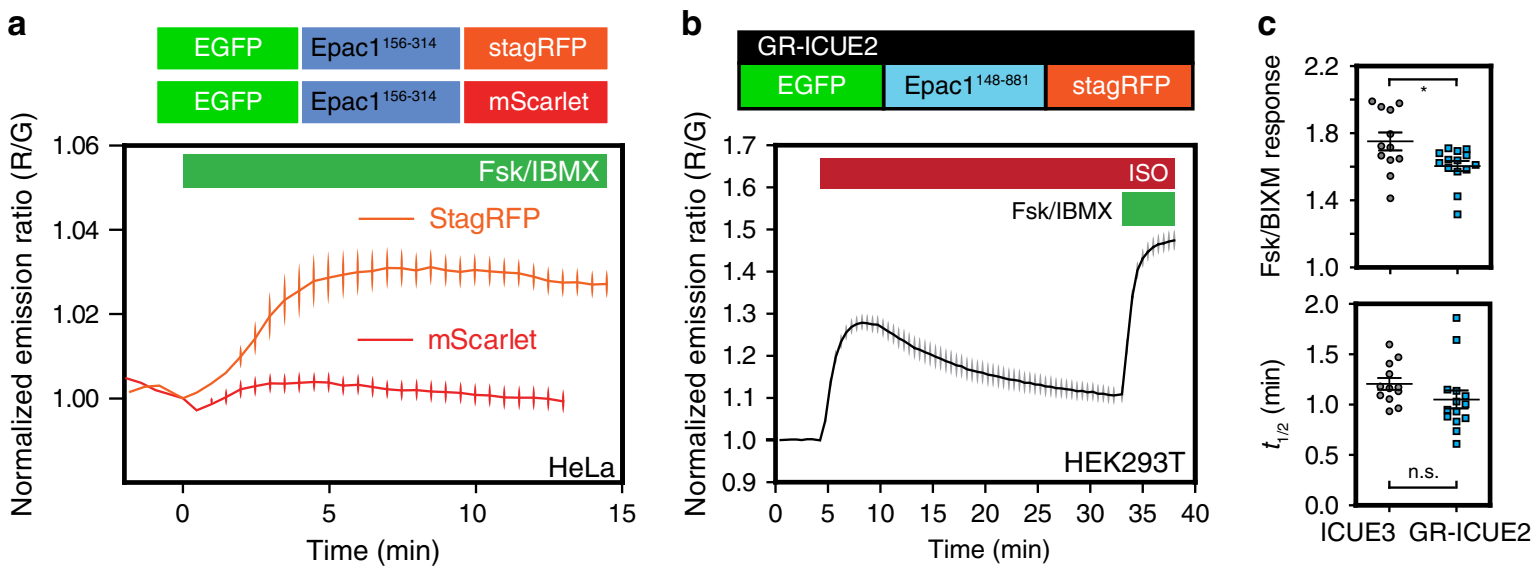

Fig. 3 The cAMP detection capability of FRET biosensors using stagRFP is comparable to cyan-yellow biosensors. a Epac1-camps biosensor is utilized to demonstrate the advantage of stagRFP, where the same sensor design yields a viable FRET biosensor using stagRFP, but not using the ultra-bright RFP $\mathrm{mScarlet}$. The change in normalized emission ratio (red/green) upon $50 \mu \mathrm{M}$ forskolin and $100 \mu \mathrm{M}$ IBMX stimulation is shown (stagRFP, $n=8$ biologically independent cells; $\mathrm{mScarlet}, n=11$ ). The mean ratio $\pm \mathrm{SEM}$ is displayed. $\mathbf{b}$ The response to adrenergic stimulation (1 $\mu \mathrm{M}$ isoproterenol) in HEK293T cells is captured by GR-ICUE2 at high dynamic range. The change in normalized emission ratio (green/red) is shown ( $n=20$ ). The mean ratio \pm SEM is displayed. c The dynamic range of GR-ICUE2 is comparable to, but still significantly smaller ( ${ }^{*} p=0.03, t=2.399$, df $=17$, from two tailed, unpaired Welch's $t$-test) than the best cyan-yellow cAMP biosensor ICUE3; upon Fsk and IBMX stimulation, the kinetics of GR-ICUE2 are not significantly different from those of ICUE3 (ICUE3, $n=12$; GR-ICUE2, $n=14$ ). The mean ratio \pm SEM is displayed, and $n$. $\mathrm{s}$. is not significant. Source data for $\mathbf{c}$ is provided as a Source Data file.

In HEK293T cells, the new fRR-EKARev biosensor displays an average response of $15.1 \pm 0.6 \%(n=12$, Fig. $4 a)$ after human EGF (huEGF) stimulation $(100 \mathrm{ng} / \mathrm{mL})$; HEK293T cells pretreated with the MEK inhibitor U0126 $(20 \mu \mathrm{M})$ showed little huEGF stimulated ERK response. The response of the new fRREKARev biosensor can also be detected as a decrease in the donor (stagRFP) fluorescence lifetime using FLIM (Supplementary Fig. 4) or as an apparent decrease in the acceptor fluorescence anisotropy using anisotropy imaging (Supplementary Fig. 5). These data demonstrate that stagRFP and smURFP form an efficient FRET pair. In brief, our data across different biosensing scenarios suggest that stagRFP is a highly effective FRET acceptor or donor and a useful alternative to current, bright RFPs.

stagRFP supports multiplexed signaling dissection. To comprehensively decipher complex signal transduction pathways in living cells, we need to monitor not just the leading actor, but also a supporting cast of enzymes both upstream and downstream of a signaling node. Expanding the spectral range of FRET-based biosensors allows us to directly increase the number of biochemical activities one can multiplex and simultaneously observe. Biochemical experiments have shown that platelet-derived growth factor (PDGF) stimulation initiates a complex signaling cascade in NIH 3T3 fibroblasts involving Src, Akt, and ERK, which are nodal points of distinct survival/growth pathways. While these kinase activities have been monitored individually ${ }^{18-20}$, simultaneously imaging of all three has not been possible to observe the relative temporal hierarchy of triple kinase cascades in real time within the same cell. To address this question, we set out to test the multiplexed imaging of 3 different biochemical activities using fRR-EKARev with other stagRFP-bearing FRET biosensors within the same living cells.

We constructed new variants of the Akt activity reporter (AktAR) and Src activity reporter (SrcAR) biosensors utilizing spectrally orthogonal donors Venus and T-sapphire, respectively, with stagRFP as the shared acceptor (Fig. 4b, c) for co-imaging ${ }^{21}$. Both of these new biosensors performed as expected based on previous use of these molecular switches in single-construct FRET imaging (Fig. 4b, c and Supplementary Fig. 6). The above mentioned fRR-EKARev was utilized to monitor ERK. Using a sequential acquisition scheme, these probes allowed us to follow the complex kinase kinetic activation relationships in single living cells. In single NIH 3T3 cells, PDGF stimulation elicited a rapid response from Src, followed by Akt, and then ERK (Fig. 4d). These multiplexed observations were consistent with their equivalent from single biosensor experiments (Supplementary Fig. 6). We estimated that the onset of Src activity occurred on average $1.0 \pm 0.6 \mathrm{~min}(n=20)$ after PDGF stimulation, whereas the beginning of Akt and ERK kinase activity was registered to $3.4 \pm 2.1 \mathrm{~min}(n=21)$ and $3.4 \pm 2.2 \mathrm{~min}(n=11)$ after stimulation, respectively (Supplementary Fig. 7A; individual curves in Supplementary Fig. 8). The kinetics of Src activity rise is significantly faster on average $\left(t_{1 / 2}=4.0 \pm 2.3 \mathrm{~min}, n=20 ; p=\right.$ $0.005, t=2.948, \mathrm{df}=38.35)$ than those of Akt $\left(t_{1 / 2}=6.3 \pm 2.8\right.$ $\min , n=21)$. The kinetics of ERK activity rise were more variable in these cells $\left(t_{1 / 2}=6.3 \pm 3.0 \mathrm{~min}, n=11\right)$, and many cells did not show a strong ERK response (Supplementary Fig. 8). Using this multiplex platform, we showed that this fast-cascade system is hampered by selective Src family kinase inhibitors, PP2 and Src11, prior to PDGF stimulation (Fig. 4d; individual curves in Supplementary Figs. 9 and 10). Under these conditions, Akt and ERK activities were markedly slower and, in some cases, largely dampened in amplitude. This hints at a built-in hierarchy to PDGFR signaling in NIH 3T3 cells. While making these observations using separate biosensors in different cells, cell-cell variations in receptor and kinase expression would introduce population-level error and mask the kinetic and temporal relationship between these kinases. Our multiplexing approach removes such ambiguity and directly examines the inter-kinase activity relationship as they occur in each single cell, demonstrating a more holistic platform for deciphering complex signaling cascades.

\section{Discussion}

In this work, we utilized a single rational mutation to enhance TagRFP-T, leading to the development of stagRFP. The successful D159V mutation is directly C-terminal to the S158T mutation in TagRFP-T ${ }^{2}$, but on the external face of the $\beta$-barrel. We surmise that in TagRFP and TagRFP-T, D159 acts as a charged "lever" to the internal environment, permitting the $\beta$-barrel and 
a
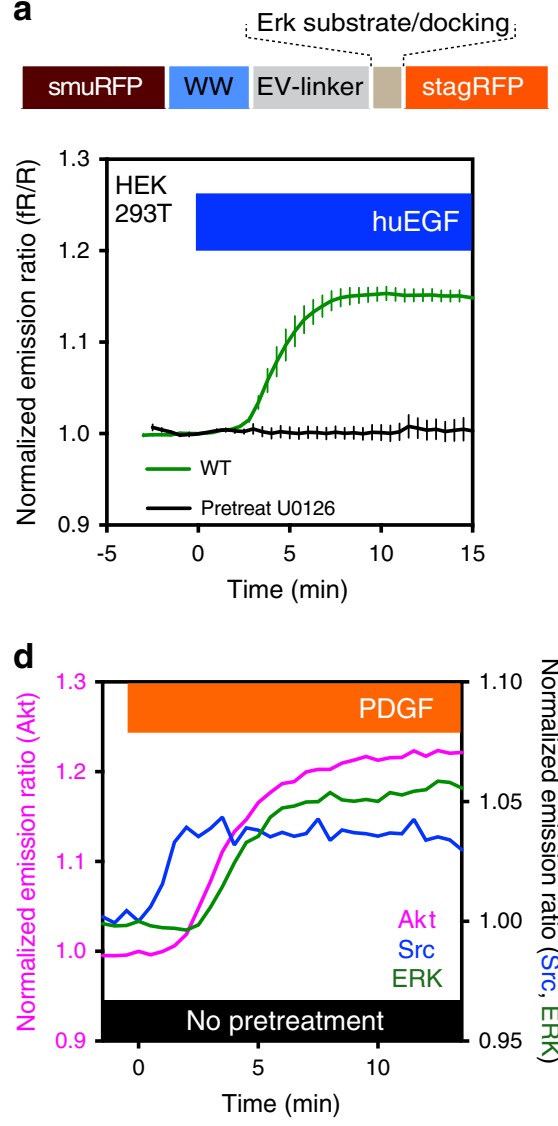

b
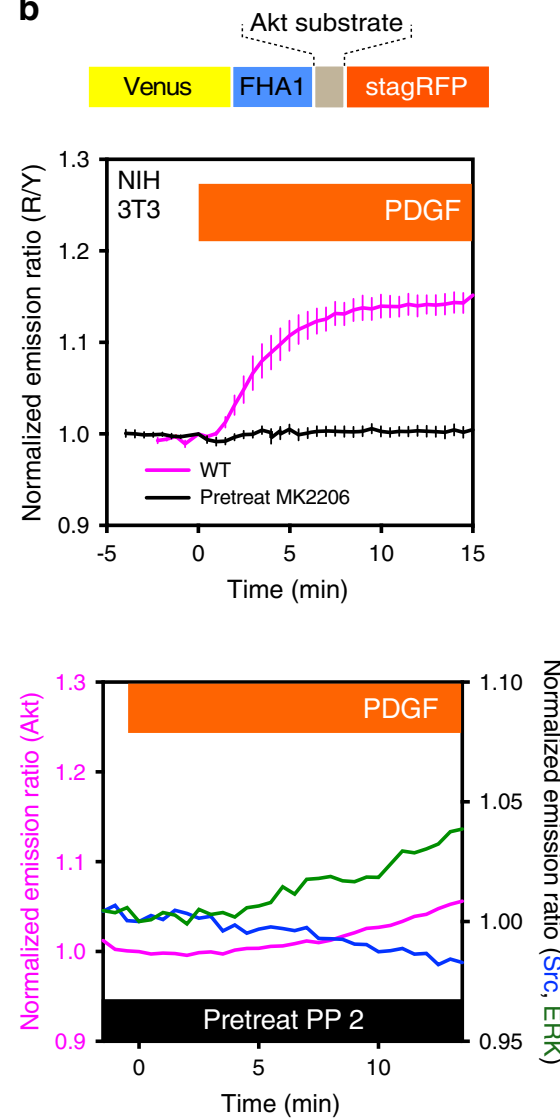

C
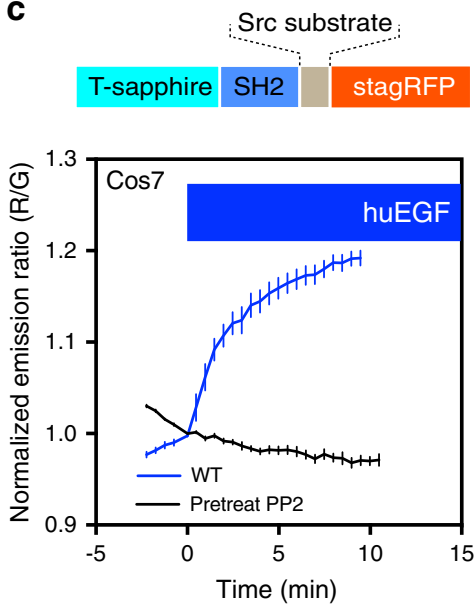

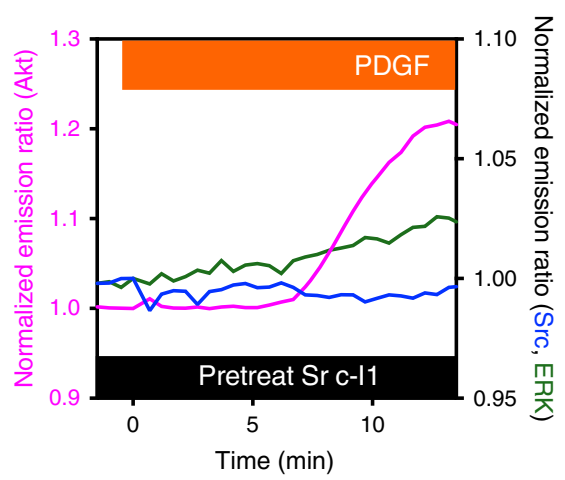

Fig. 4 Multiplexing the kinase activities of Akt, Src, and ERK in single live cells utilizing three biosensors containing stagRFP. a The red-far-red biosensor fRR-EKARev could reliably monitor ERK activity in response to $100 \mathrm{ng} / \mathrm{mL}$ huEGF stimulation in the red-far-red region, shown as changes in normalized emission ratio (far-red/red) (WT, $n=12$ biologically independent cells; and pretreatment with $20 \mu M \cup 0126, n=5$ ). b The yellow-red Akt activity reporter incorporating stagRFP reliably detects Akt activity in NIH 3 T3 cells in response to $50 \mathrm{ng} / \mathrm{mL}$ PDGF stimulation, shown as changes in normalized emission ratio (red/yellow) (WT, $n=6$; and pretreatment with $3 \mu$ M MK2206, $n=8$ ). c The green-red Src activity reporter incorporating stagRFP reliably detects Src activity in Cos7 cells in response to $100 \mathrm{ng} / \mathrm{mL}$ huEGF stimulation, shown as changes in normalized ratio (red/green) (green for T-sapphire emission) (WT, $n=6$; and pretreatment with $10 \mu \mathrm{M} \mathrm{PP2}, n=12$ ). The mean ratio \pm SEM is displayed for a-c. $\mathbf{d}$ Multiplexed imaging revealed a potential kinetic relationship between Src, Akt, and ERK upon $50 \mathrm{ng} / \mathrm{mL}$ PDGF stimulation. Representative curves showing PDGF-induced responses of green-red based Src activity biosensor (blue), yellow-red based Akt activity biosensor (red), and ERK activity biosensor (green) in control cells without pretreatment (left), cells pretreated with $10 \mu \mathrm{M}$ PP2 (middle), or $10 \mu \mathrm{M}$ Src-11 (right).

chromophore in turn to be perturbed externally by solvent and protein-protein interactions. $\mathrm{D} 159 \mathrm{~V}$ is a novel example of an external residue that directly contributes to chromophore function. Beyond charge neutralization, the valine modification may provide hydrophobic spatial bulk and limit hydrophilic solvent accessibility to the chromophore. The engineering considerations and insights, reported here, should inform the study of similar residues on other $\beta$-barrel fluorescent proteins, to potentially reveal previously overlooked properties.

We also developed a multiplexed imaging approach for analyzing three signaling activities in single living cells. Quantitative temporal relationship between different signaling events provides critical insights into signal propagation along a signaling cascade or dynamic cross-regulation between different pathways. However, this information is difficult to obtain from population-based biochemical assays owing to their limited temporal resolution. On the other hand, monitoring one signaling activity at a time in single-cell imaging and analyzing temporal correlation from parallel experiments can also present challenges because of cellto-cell variations arising from differences in the expression of critical signaling components, such as membrane receptors. Multiplexed imaging simultaneously captures the precise temporal relationship between multiple signaling activities in a single living cell and provides a valuable tool to investigate the complex interplays.

Our visualization of the triple kinase activities following PDGFR stimulation in living NIH 3T3 cells illustrates the complexity of receptor signaling and suggests a cascade potentiated by Src family kinases, where several signaling branches, such as Akt/ mTORC1 and Ras/MEK/ERK, may be modulated by these nonreceptor tyrosine kinases. The relationships between Src and these branches have been reported but not directly interrogated simultaneously in the same cells. For example, Src was shown to mediate hormone (such as estrogen) signaling by directly activating PI3K/Akt through phosphorylation of the PI3K regulatory subunit $\mathrm{p} 85^{22,23}$. Src family kinases can also phosphorylate Grb2associated-binders (Gab) family members, providing binding sites for multiple effector proteins, such as Src homology-2 (SH2)containing protein tyrosine phosphatase 2 (SHP2) and p85. Although the interaction between Ras/Raf and Src is multilayered $^{24}$, Src can directly phosphorylate Raf to activate downstream MAP kinases, such as ERK ${ }^{25,26}$. Using a relatively selective inhibitor for Src family kinases PP2 27,28 and an alternative dual competitive inhibitor for ATP and the substrate binding sites Src Inhibitor-1 (Src-I1) $)^{29,30}$, we show that Src family kinases significantly modulate the landscape of downstream kinases in 
PDGFR signaling. Future work will further dissect the underlying mechanisms and analyze the temporal activation to examine whether, similar to EGF/NGF signaling in PC12 cells $^{31}$, NIH 3T3 fibroblasts can also actively encode distinct receptor signaling through kinetic kinase activation.

The multiplexed imaging method, demonstrated here, is complementary to other approaches, such as homoFRET anisotropy imaging and single-color (intensity-based) biosensing, each with associated benefits and disadvantages. HomoFRET detection requires specialized polarization optics and analysis and has limited dynamic range, but provides very rapid kinetics ${ }^{32}$. Singlecolor intensity sensors are laborious to develop and frequently sensitive to unintended responses, such as $\mathrm{pH}$, but require the simplest microscopy instrumentation and regularly provide the largest dynamic range. In comparison, our development of stagRFP enhances quantitative ratiometric FRET imaging, making stagRFP generally applicable to any combination of FRET biosensors without the extensive optimization required for singlecolor sensors. Our data suggest that stagRFP is a useful FRET donor and acceptor, making stagRFP a crucial addition to the genetically encoded FP FRET toolkit. In the absence of such an RFP, biosensing is limited to utilizing multiple RFP species where inseparable cross-talk contaminates all FRET readouts involved. The TagRFP chromophore has a large 2-photon absorption crosssection at a region where its one-photon extinction is low $^{33}$, making it a useful marker in 2-photon fluorescence imaging. The spectral similarity between stagRFP and its parent FP suggests that this characteristic could have been retained. Future work will examine 2-photon properties of stagRFP both in vitro and in cells, followed by 2-photon imaging of fRR-EKARev in cells and tissues. We expect that the new red-far-red FRET pair we developed should facilitate studies of the spatiotemporal activation of ERK and other kinases in vivo. In conclusion, we have demonstrated the rational improvement of TagRFP-T to create the bright stagRFP and showcased the improvements stagRFP contributes to FRET biosensors and multiplex imaging. The prevalence of many existing FRET molecular switches permits new sets of biochemical activities to be multiplexed simply by incorporating the FP pairings we reported here.

\section{Methods}

Mutagenesis. All site-directed mutagenesis experiments were performed following the protocol of Sawano and Miyawaki ${ }^{34}$ using the E. coli DH5a strain and plated onto LB-Amp agar. Random mutagenic primers were fully degenerate at the R157, D159, D196, and R198 residues. The TagRFP-T mutants were screened by colony fluorescence and only brightly fluorescent colonies (in both GFP and RFP channels) were sequenced. The mean intensity of each candidate in the RFP color channel was measured using an in-house fluorescence imager, illuminated by a broad-spectrum lamp source (MAX-303, Asahi Spectra) and monitored by a Thorlabs USB digital camera mounted behind a Thorlabs emission filter wheel for different fluorescence colors (CFP: 430/40; GFP: 535/40; and RFP: 630/75 nm). The images were analyzed using Image (v. $1.47 \mathrm{~g}$ or later).

Protein purification and characterization. TagRFP-T and stagRFP were overexpressed in the JM109 strain using pRSET-B vector at $37^{\circ} \mathrm{C}$ then subsequently purified via His-tag affinity chromatography on separate Ni-NTA columns (Qiagen). The proteins were eluted using an imidazole gradient in $20 \mathrm{mM}$ increments up to $200 \mathrm{mM}$ imidazole. Pure fractions were concentrated via Amicon Ultra-15 filters $(10,000 \mathrm{NMWL})$, stored at $4{ }^{\circ} \mathrm{C}$ in $50 \mathrm{mM}$ Tris buffer with $300 \mathrm{mM} \mathrm{NaCl}$ at $\mathrm{pH} 7.4$, and characterized within one month. Semi-native PAGE of stagRFP was performed using $10 \%$ polyacrylamide gels on non-boiled protein samples with minimal SDS content and stained using Coomassie Blue. Purified fluorescent proteins were simultaneously characterized by absorption (Beckman DU-650 spectrophotometer) and fluorescence (Horiba Jobin Yvon FluoroMax-3 fluorimeter). The absorbance spectra were normalized to the respective absorbance at $280 \mathrm{~nm}$ and the fluorescence spectra were normalized to the fluorescence maximum of TagRFP-T.

Biosensor cloning. Cloning and subcloning were performed using the DH5a strain of $E$. coli. All mammalian constructs were cloned into the pcDNA3.0 vector with a modified multiple cloning site and all bacterial constructs were cloned into the pRSET-B vector. Plasmids were generated as follows: For GR-AKAR3 and variants, biosensor inserts were first subcloned into the pRSET-B vector using the restriction enzyme (RE) sites BamHI and EcoRI; the C-terminal fluorescent protein was then replaced by a PCR amplified stagRFP or mScarlet using primers encoding a 5' SacI site and a 3' EcoRI site; the donor position was then replaced by a PCR amplified EGFP using primers encoding a 5' BamHI site and a 3' BglII site. The green-red biosensor was subcloned into the pcDNA3.0 vector. Subsequent new biosensors (AktAR, SrcAR, and EKAR) employing the desired green-red FRET pairing were generated by replacing the AKAR biosensors with the target green-red pair using a PCR amplified fragment of the sensing unit alone using primers encoding a 5' BglII site and a 3' SacI site while in the context of pRSET-B, and subsequently subcloned into the pcDNA3.0 vector. In cases where these RE sites were not unique, site directed mutagenesis was utilized to obtain a unique combination or other RE sites, such as KpnI, were encoded instead. fRR-EKARev was generated by following a very similar protocol but placing smURFP at the N-terminal fluorescent protein position. Primer sequences are in Supplementary Table 1

OSER assay. pCytERM-mScarlet was a gift from Dorus Gadella (85066, Addgene) and CytERM-stagRFP was created by replacing the mScarlet with a PCR amplified stagRFP using primers encoding a 5' AgeI site and a 3' NotI site. OSER assay imaging was performed on a Nikon A1 confocal microscope. Hela cells at $\sim 70 \%$ confluency were transfected with $500 \mathrm{ng}$ of CytERM-FP constructs using Lipofectamine 2000 and incubated for $24 \mathrm{~h}$ before imaging. ImageJ was used to measure mean fluorescence intensity (MFI) of each OSER structure. Each cell nuclear envelope (NE) MFI was averaged from three MFI measurements from different edges of the nuclei, excluding any karmellae regions. Cells containing lobed or indistinct nuclei were excluded.

Cell culture. The HeLa, HEK293T, and NIH 3T3 cells were maintained in DMEM media supplemented with $10 \%$ FBS and $1 \%$ penicillin and streptomycin. All cells were transfected at $\sim 70 \%$ confluency using Lipofectamine 2000 and incubated for $24 \mathrm{~h}$ before imaging. NIH 3T3 cells were serum-starved to optimize growth-factor stimulation. Pretreatment of cells, to validate kinase biosensors, was performed by incubating cells in HBSS buffer with inhibitors at $37^{\circ} \mathrm{C}$ for $15 \mathrm{~min}$ prior to imaging at the following concentrations. For ERK: HEK293T cells were treated with $20 \mu \mathrm{M}$ U0126; for Akt: NIH3T3 cells were treated with $3 \mu \mathrm{M}$ MK2206; for Src: Cos7 cells were treated with $10 \mu \mathrm{M}$ PP2; and NIH 3T3 cells with $10 \mu \mathrm{M}$ PP2 or Src-I1. All cells were imaged in HBSS buffer at room temperature. Cells transfected with fRREKARev biosensor were incubated with $12.5 \mu \mathrm{M}$ biliverdin for $30 \mathrm{~min}$ in HBSS buffer at $37^{\circ} \mathrm{C}$ before imaging.

Fluorescence microscopy. All epifluorescence imaging was performed on a Zeiss Axiovert 200 M, AxioObserver Z1 Microscope, or AxioObserver Z7 equipped with a xeon lamp, a Sutter Lambda 10-3 that controls two separate filter wheels, a cooled (or cooled electron-multiplying) CCD, and examined under a 40X oil immersion objective. FRET microscopy of GFP/RFP biosensors was performed using the following excitation (EX) and emission (EM) filter combinations (max$\mathrm{ima} /$ bandwidth in nm): GFP-EX 480/30, EM 535/45, D505; RFP-EX 568/55, EM 653/95, D600; and FRET: EX 480/30, EM 653/95, D505. Emission intensities of individual cells were background-subtracted, and the ratios between the donor and FRET channel were normalized to the timepoint just before addition of stimuli $(t=0 \mathrm{~min})$

Multiplex imaging. Co-imaging of 3 FRET biosensors was performed using either Multiplexed Filter Set A or Multiplexed Filter Set B. Multiplexed Filter Set A contains the following filter combinations (maxima/bandwidth in $\mathrm{nm}$, dichroic (D) cutoff in nm): T-sapphire-RFP FRET-EX 380/10, EM 653/95, and D505; YFP-RFP FRET-EX 495/10, EM 653/95, and D515; smURFP-RFP FRET-EX 555/25, EM 700/75, and D660; RFP-EX 555/25, EM 653/95, and D600; T-sapphire-EX 380/10, EM 535/45, and D505; and YFP-EX 495/10, EM 535/25, and D515. Multiplexed Filter Set B contains the following filter combinations (maxima/bandwidth in $\mathrm{nm}$, dichroic (D) cutoff in nm): T-sapphire-RFP FRET-EX 380/10, EM 645/75, and D505; YFP-RFP FRET-EX 495/10, EM 645/75, and D515; smURFP-RFP FRET-EX 572/35, EM 700/75, and D594; RFP-EX 572/35, EM 653/95, and D594; T-sapphire-EX 380/10, EM 535/50, and D505; and YFP-EX 495/10, EM 535/25, and D515. All FRET data was acquired using MetaFluor software and subsequently analyzed using MetaFluor software or in-house ImageJ macro programs and MATLAB scripts.

Photoactivation studies. To enable photoactivation studies, cyclic excitation imaging was performed under total-internal-reflection fluorescence (TIRF) conditions using HeLa cells expressing plasma membrane-targeted RFP on a Nikon NSTORM/TIRF microscope equipped with an Andor IXON3 Ultra DU897 EMCCD camera, a Melles Griot argon laser (green-red FRET excitation: $488 \mathrm{~nm}$ ), and a Coherent Sapphire solid-state laser (RFP direct excitation: $561 \mathrm{~nm}$ ). Green-red FRET and then RFP direct images were taken at $100 \mathrm{~ms}$ exposures every $45 \mathrm{~s}$ with gain of 100, 10\% setting on $488 \mathrm{~nm}$ power, and 1\% setting on $561 \mathrm{~nm}$ power. 
Fluorescent lifetime imaging microscopy. FLIM was performed at 100× magnification on a Leica SP8 FALCON confocal microscope equipped with a white light laser and a Lecia HyD SMD detector. The laser was tuned to $500-530 \mathrm{~nm}$ for excitation of GR-AKAR-3 and tuned to $570-610 \mathrm{~nm}$ for excitation of fRR-EKARev and pulsed at a frequency of $80 \mathrm{MHz}$. Time course images were acquired every 2.5 min. Intensity weighted mean fluorescence lifetimes were calculated in LAS X (Lecia) software by fitting a bi-exponential decay model to the decay of each ROI. One ROI was selected per cell. Hela cells transfected with fRR-EKARev were serum starved overnight and incubated with HBSS and $12.5 \mu \mathrm{M}$ biliverdin at room temperature for $30 \mathrm{~min}$ before performing FLIM.

Anisotropy microscopy. Fluorescence anisotropy imaging was performed using a $\times 200.45$ NA objective lens on a Zeiss AxioObserver. A polarizer (Chroma) was placed in the excitation pathway between the xenon arclamp and the excitation filters. Emission polarizations were separated using an Opto-Split II LS image splitter, with two wire grid polarizers (Meadowlark) oriented perpendicular and parallel to the excitation polarizer. The Hamamatsu Flash 4.0 sCMOS camera collected images containing both polarizations. FRET microscopy of GR-AKAR-3 and fRR-EKARev were performed using the following excitation (EX) and emission (EM) filter combinations (maxima/bandwidth in nm): GR-AKAR-3 FRET: EX 480/ 30, EM 653/95, D505 and fRR-EKARev FRET: EX 568/55, EM 700/75, D600.

Image analysis was performed using Fiji (ImageJ). Polarization images were cropped, background subtracted using Fiji's built-in Rolling Ball Background Subtraction function, and then aligned using Fiji's built-in StackReg function. Regions of Interest (ROIs) were manually selected in Fiji and their fluorescence emission intensities in both polarizations were measured. The anisotropy of each ROI was calculated using the equation,

$$
r=\frac{I_{\|}-g \cdot I_{\perp}}{I_{\|}+2 \cdot g \cdot I_{\perp}}
$$

where $I_{\|}$and $I_{\perp}$ are the background subtracted fluorescence intensities in the parallel and perpendicular polarizations, respectively, and $g$ is the correction factor accounting for differences in polarization emission efficiencies within the instrument. The g-factor was calculated using an isotropic fluorescein solution ${ }^{35}$. The change in anisotropy for each timepoint was calculated by subtracting the anisotropy value at the timepoint right before drug stimulation from the anisotropy calculated for that timepoint. Hela cells were transfected at $\sim 70 \%$ confluency using Lipofectamine 2000 one day before imaging. Hela cells transfected with fRREKARev were serum starved overnight and incubated with HBSS and $12.5 \mu \mathrm{M}$ biliverdin at $37^{\circ} \mathrm{C}$ for $30 \mathrm{~min}$ before performing anisotropy imaging.

Statistical methods. The reported statistical significance between control and experimental datasets were the results of two tailed, unpaired Welch's $t$-tests calculated at 95\% confidence level using Graphpad Prism.

Reporting summary. Further information on research design is available in the Nature Research Reporting Summary linked to this article.

\section{Data availability}

The source data underlying visible spectra and bar graphs in Figs. 1-3 and Supplementary Figs. 1 and 7 are provided as a source data file. The plasmids utilized in this article will be submitted to the non-profit plasmid repository, Addgene, for scientific sharing. All other data supporting the findings of this study, including the MetaFluor pseudocode/protocol are available upon reasonable request.

Received: 23 July 2019; Accepted: 20 March 2020;

Published online: 15 April 2020

\section{References}

1. Merzlyak, E. M. et al. Bright monomeric red fluorescent protein with an extended fluorescence lifetime. Nat. Methods 4, 555-557 (2007).

2. Shaner, N. C. et al. Improving the photostability of bright monomeric orange and red fluorescent proteins. Nat. Methods 5, 545-551 (2008).

3. Dean, K. M. et al. Analysis of red-fluorescent proteins provides insight into dark-state conversion and photodegradation. Biophys. J. 101, 961-969 (2011).

4. Mo, G. C. H. et al. Genetically encoded biosensors for visualizing live-cell biochemical activity at super-resolution. Nat. Methods 14, 427-434 (2017).

5. Costantini, L. M., Fossati, M., Francolini, M. \& Snapp, E. L. Assessing the tendency of fluorescent proteins to oligomerize under physiologic conditions. Traffic 13, 643-649 (2012).

6. Cranfill, P. J. et al. Quantitative assessment of fluorescent proteins. Nat. Methods 13, 557-562 (2016).
7. Zhang, J., Ma, Y., Taylor, S. S. \& Tsien, R. Y. Genetically encoded reporters of protein kinase A activity reveal impact of substrate tethering. Proc. Natl Acad. Sci. USA 98, 14997-15002 (2001).

8. Depry, C., Allen, M. D. \& Zhang, J. Visualization of PKA activity in plasma membrane microdomains. Mol. Biosyst. 7, 52-58 (2011).

9. Komatsu, N. et al. Development of an optimized backbone of FRET biosensors for kinases and GTPases. Mol. Biol. Cell 22, 4647-4656 (2011).

10. Lam, A. J. et al. Improving FRET dynamic range with bright green and red fluorescent proteins. Nat. Methods 9, 1005-1012 (2012).

11. Bindels, D. S. et al. mScarlet: a bright monomeric red fluorescent protein for cellular imaging. Nat. Methods 14, 53-56 (2017).

12. Nikolaev, V. O., Bünemann, M., Hein, L., Hannawacker, A. \& Lohse, M. J. Novel single chain cAMP sensors for receptor-induced signal propagation. $J$. Biol. Chem. 279, 37215-37218 (2004).

13. Klarenbeek, J., Goedhart, J., Van Batenburg, A., Groenewald, D. \& Jalink, K. Fourth-generation Epac-based FRET sensors for cAMP feature exceptional brightness, photostability and dynamic range: characterization of dedicated sensors for FLIM, for ratiometry and with high affinity. PLOS ONE 10, e0122513 (2015).

14. DiPilato, L. M., Cheng, X. \& Zhang, J. Fluorescent indicators of cAMP and Epac activation reveal differential dynamics of cAMP signaling within discrete subcellular compartments. Proc. Natl Acad. Sci. USA 101, 16513-16518 (2004).

15. Dipilato, L. M. \& Zhang, J. The role of membrane microdomains in shaping beta2-adrenergic receptor-mediated cAMP dynamics. Mol. Biosyst. 5, 832-837 (2009).

16. Shcherbakova, D. M., Cammer, N. C., Huisman, T. M., Verkhusha, V. V. \& Hodgson, L. Direct multiplex imaging and optogenetics of Rho GTPases enabled by near-infrared FRET. Nat. Chem. Biol. 14, 591-600 (2018).

17. Rodriguez, E. A. et al. A far-red fluorescent protein evolved from a cyanobacterial phycobiliprotein. Nat. Methods 13, 763-769 (2016).

18. Tang, S. \& Yasuda, R. Imaging ERK and PKA activation in single dendritic spines during structural plasticity. Neuron 93, 1315-1324.e3 (2017).

19. Wang, Y. et al. Visualizing the mechanical activation of Src. Nature 434, 1040-1045 (2005).

20. Zhou, X. et al. Dynamic visualization of mTORC1 activity in living cells. Cell Rep. 10, 1767-1777 (2015).

21. Aye-Han, N.-N., Allen, M. D., Ni, Q. \& Zhang, J. Parallel tracking of cAMP and PKA signaling dynamics in living cells with FRET-based fluorescent biosensors. Mol. Biosyst. 8, 1435-1440 (2012).

22. Cuevas, B. D. et al. Tyrosine phosphorylation of $\mathrm{p} 85$ relieves its inhibitory activity on phosphatidylinositol 3-kinase. J. Biol. Chem. 276, 27455-27461 (2001).

23. Haynes, M. P. et al. Src kinase mediates phosphatidylinositol 3-kinase/Aktdependent rapid endothelial nitric-oxide synthase activation by estrogen. $J$. Biol. Chem. 278, 2118-2123 (2003).

24. Bunda, S. et al. Src promotes GTPase activity of Ras via tyrosine 32 phosphorylation. Proc. Natl Acad. Sci. USA 111, E3785-E3794 (2014).

25. Marais, R., Light, Y., Fpaterson, H. \& Marshall, C. J. Ras recruits Raf-1 to the plasma membrane for activation by tyrosine phosphorylation. EMBO J. 14, 3136-3145 (1995).

26. Ziogas, A., Moelling, K. \& Radziwill, G. CNK1 is a scaffold protein that regulates Src-mediated Raf-1 activation. J. Biol. Chem. 280, 24205-24211 (2005).

27. Hanke, J. H. et al. Discovery of a novel, potent, and Src family-selective tyrosine kinase inhibitor: study of Lck- and FynT-dependent T cell activation. J. Biol. Chem. 271, 695-701 (1996).

28. Karni, R. et al. The pp60c-Src inhibitor PP1 is non-competitive against ATP. FEBS Lett. 537, 47-52 (2003).

29. Tian, G., Cory, M., Smith, A. A. \& Knight, W. B. Structural determinants for potent, selective dual site inhibition of human pp60 c-src by 4anilinoquinazolines. Biochemistry 40, 7084-7091 (2001).

30. Bain, J. et al. The selectivity of protein kinase inhibitors: a further update. Biochem. J. 408, 297-315 (2007).

31. Ryu, H. et al. Frequency modulation of ERK activation dynamics rewires cell fate. Mol. Syst. Biol. 11, 838-838 (2015).

32. Ross, B. L. et al. Single-color, ratiometric biosensors for detecting signaling activities in live cells. Elife 7, e35458 (2018).

33. Drobizhev, M., Makarov, N. S., Tillo, S. E., Hughes, T. E. \& Rebane, A. Twophoton absorption properties of fluorescent proteins. Nat. Methods 8, 393-399 (2011).

34. Sawano, A. \& Miyawaki, A. Directed evolution of green fluorescent protein by a new versatile PCR strategy for site-directed and semi-random mutagenesis. Nucleic Acids Res. 28, e78 (2000).

35. Piston, D. W. \& Rizzo, M. A. FRET by fluorescence polarization microscopy. Methods Cell Biol. 85, 415-430 (2008). 


\section{Acknowledgements}

We thank Professor Roger Tsien for connecting the development of the bright far-red FP smURFP to our work on the new RFP, sharing constructs, and discussing the location of the mutation. We also thank Sohum Mehta for critical reading of the manuscript. We thank Brian Tenner for training in anisotropy imaging. This work was supported by R35 CA197622, R01 DK073368, and R01 GM111665 to J.Z. C.P. was supported by NIH Grant T32 GM008326. Confocal imaging was done at the UC San Diego Nikon Imaging Center with the assistance of Dr. Eric Griffis. FLIM imaging was done at the UC San Diego School of Medicine Microscopy Core, funded by NS047101 grant.

\section{Author contributions}

G.M. and J.Z. conceived the idea. G.M., C.P., and J.Z. designed all experiments. G.M. performed the mutagenesis, purification, spectroscopy, and biosensor construction. E.A.R. provided the gene for the unpublished fluorescent protein, smURFP. G.M. and C.P. performed all microscopy except FLIM, which was performed by T.S. G.M., C.P. and T.S. analyzed the microscopy data. G.M., C.P., and J.Z. wrote the paper with critical inputs from E.A.R.

\section{Competing interests}

The authors declare no competing interests.

\section{Additional information}

Supplementary information is available for this paper at https://doi.org/10.1038/s41467020-15687-x.

\section{Correspondence and requests for materials should be addressed to J.Z.}

Peer review information Nature Communications thanks Jean-François Bodart and the other, anonymous, reviewer(s) for their contribution to the peer review of this work.

Reprints and permission information is available at http://www.nature.com/reprints

Publisher's note Springer Nature remains neutral with regard to jurisdictional claims in published maps and institutional affiliations.

(c) (i) Open Access This article is licensed under a Creative Commons Attribution 4.0 International License, which permits use, sharing, adaptation, distribution and reproduction in any medium or format, as long as you give appropriate credit to the original author(s) and the source, provide a link to the Creative Commons license, and indicate if changes were made. The images or other third party material in this article are included in the article's Creative Commons license, unless indicated otherwise in a credit line to the material. If material is not included in the article's Creative Commons license and your intended use is not permitted by statutory regulation or exceeds the permitted use, you will need to obtain permission directly from the copyright holder. To view a copy of this license, visit http://creativecommons.org/ licenses/by/4.0/.

(C) The Author(s) 2020 